\title{
O PLANEJAMENTO E A ESTIMATIVA DE CUSTO NO AMBIENTE DE MODELAGEM BIM: UM ESTUDO DE CASO
}

\author{
(1) INBEC, Fortaleza \\ (2) Quatre Ensino Especializado, Natal
}

Carlos Renato da Mota Bezerra ${ }^{(1)}$, Sandra Albino Ribeiro ${ }^{(2)}$

\begin{abstract}
Resumo
No Brasil, é crescente o número de profissionais da área de Arquitetura, Engenharia e Construção Civil (AEC) que estão aderindo ao Building Information Modelling (BIM). Isso vem ocorrendo pelo entendimento de que essa metodologia traz inúmeros benefícios. Por outro lado, ressalta-se que o BIM, apesar de possuir um aporte teórico amplo, também se mostra complexo e apresenta algumas lacunas, tornando necessário o desenvolvimento de ferramentas, técnicas e diretrizes para seu melhor uso. Nesse sentido, este trabalho teve por objetivo descrever e analisar a aplicação de ferramentas BIM no processo de orçamentação e planejamento de obras. A pesquisa é descritiva e ocorreu através do estudo de caso. Dentre os resultados obtidos, destaca-se que o processo em BIM possibilitou a visualização da obra em várias fases de execução, uma maior acurácia na quantificação, a elaboração da estimativa de custo no próprio ambiente de modelagem, a geração de um cronograma assertivo, além de ganhos potenciais frente à simulação tridimensional, a qual facilita a visualização e compreensão das etapas de serviços. Embora haja diversos ganhos, alerta-se para o empenho no desenvolvimento de modelagens com alto nível de detalhamento e o uso da programação como meio para ampliar a automação e a interoperabilidade.
\end{abstract}

\section{Introdução}

Mesmo com o avanço das inovações tecnológicas em diversas áreas da indústria, a construção civil ainda se mostra relativamente atrasada, utilizando técnicas rudimentares, apresentando baixos índices de produtividade e altos percentuais de desperdício em comparação a outros segmentos industriais, evidenciado pelo alto custo de produção que afeta negativamente a margem de lucro das empresas do setor [1]. Na busca por melhorar esses indicadores, o presente estudo propõe ações a serem adotadas pela indústria da construção civil no tocante ao aprimoramento dos processos de estimativa de custo e do planejamento da obra, especialmente, na programação da construção. 
Tradicionalmente, o processo de planejamento e a programação da construção utiliza diagramas de barras demonstrando o sequenciamento das atividades no espaço e no tempo. Porém esses métodos não refletem adequadamente os componentes espaciais relacionados a essas atividades, tão pouco fazem conexão com o projeto, consistindo ainda em uma tarefa manual intensa [2]. Com frequência, o produto desse processo é um plano que não está sincronizado com o projeto, que por sua vez dificulta a compreensão da programação e seu respetivo impacto na logística do canteiro, tornando ineficiente o processo de gerenciamento de obras [1].

Do outro lado, estimativas não confiáveis expõem o empreendedor a um risco considerável e majoram artificialmente os custos do projeto. A confiabilidade das estimativas de custo é afetada por diversos fatores, dentre eles, a variação das condições de mercado, atraso na execução do empreendimento, alterações no projeto, produtividade variável da mão-de-obra entre outros. Além disso, orçamentistas relatam que essa ação é quase sempre marcada pela falta de tempo para a elaboração das estimativas, indisponibilidade de informações e detalhes incipientes no projeto e falhas de comunicação entre os intervenientes, principalmente, entre o proprietário e o orçamentista [3].

Com a utilização do BIM e das suas ferramentas, o processo de elaboração de estimativas de custos e cronogramas passou a utilizar um protótipo virtual da construção cujos componentes são parametrizáveis e armazenam em si informações. Suas tabelas proporcionam uma quantificação mais rápida e eficiente, embora ainda existam limitações a serem superadas. $\mathrm{O}$ presente trabalho emergiu em meio a isso e lançou a questão "De que maneira a utilização do BIM aprimora os processos e os resultados da estimativa de custos e do planejamento de obra, especialmente, no processo de elaboração de um plano de produção? Com isso, o objetivo foi definido como: Descrever e analisar a aplicação de ferramentas BIM no processo de orçamentação e planejamento de obras e assim compreender os reais ganhos e limitações dessa nova metodologia de trabalho. Por fim, ressalta-se que esta pesquisa é oriunda e continuação de dois trabalhos de conclusão de cursos [1,3].

\section{Método}

O método adotado neste trabalho é a pesquisa descritiva que visa fazer a descrição das características de um determinado fenômeno e estabelecer relações entre variáveis. O trabalho ocorreu pelo estudo de caso, uma modalidade que busca a aplicação prática a partir do conhecimento científico. Após a definição de uma obra para o estudo de caso foi possível delinear as etapas que consistiram respetivamente: Processo tradicional, Processo BIM e Processo BIM com programação. Cada uma dessas etapas obedeceu à fluxo de trabalho que será descrito nos próximos tópicos e apresentou diferentes resultados.

\section{Estudo de caso}

O projeto utilizado neste estudo de caso trata-se de uma Habitação de Interesse Social (HIS) térrea situada em um terreno retangular de $12,00 \mathrm{~m}$ por $30,00 \mathrm{~m}$ e constituída de varanda, dois quartos, banheiro, sala de estar, cozinha e área de serviço, que perfazem $60,26 \mathrm{~m}^{2}$ de área construída. Embora seja uma edificação de pequeno porte, ela abrange uma temática atual. Vale lembrar que o déficit habitacional é um problema crônico no Brasil e atinge, 
especialmente, as famílias de baixa renda. Nesse sentido, o uso de novos processos e tecnologias apresenta potencial para minimizar custos, ampliar a produtividade e qualidade das HIS.

O projeto arquitetónico foi cedido aos pesquisadores no formato DWG e a partir desse arquivo desenvolveram a Estrutura Analítica de Projeto (EAP) e os modelos BIM de arquitetura e das instalações prediais (água fria e esgoto) no software Autodesk $®$ Revit®. Por ser uma edificação de pequeno porte, os elementos estruturais (pilares, cinta e radier) foram modelados em conjunto com a arquitetura. Os pilares estão no muro e tem $2,10 \mathrm{~m}$ de altura, as cintas de amarração tem $0,30 \mathrm{~m}$ de altura e o radier, uma laje com a função de fundação tem $0,20 \mathrm{~m}$. Antes dos processos de orçamentação e planejamento em BIM foi executado a compatibilização no software Autodesk ${ }^{\circledR}$ Navisworks ${ }^{\circledR}$, que possibilitou identificar poucas interferências entre as disciplinas modeladas. Em seguida, foram feitas correções nos modelos BIM a fim de eliminar os conflitos encontrados na etapa de compatibilização. Por fim, foi dado início ao processo de orçamentação e planejamento tradicional e em BIM.

\section{Processo tradicional}

O processo de quantificação dos componentes da edificação ocorreu por meio da leitura dos desenhos bidimensionais, contagem ou medição desses e o registo das informações em planilhas eletrônicas no Microsoft ${ }^{\circledR}$ Officie Excel ${ }^{\circledR}$ e Microsoft $₫$ MS Project ${ }^{\circledR}$. O resumo das quantidades foi organizado nesses softwares de acordo com a EAP que por sua vez subdivide o projeto em pacotes de trabalho, partes menores que podem ser bem compreendidas, planejadas e controladas. É sobre essa estrutura em que os demais processos de gerenciamento se basearam para elaborar o cronograma, orçamento, alocação de recursos. etc [1].

O preço unitário de cada pacote de trabalho ou atividade foi extraído manualmente do repositório de composições de preço unitário, encontrado na tabela oficial do SINAPI [4]. A obtenção dos custos totais foi feita em planilhas eletrônicas. O cronograma foi elaborado no Microsoft ${ }^{\circledR}$ MS Project ${ }^{\circledR}$ onde as informações sobre duração e insumos de cada atividade são inseridos manualmente. Dependendo do número de atividades, os dados inseridos nesse software referem-se apenas à mão de obra ou a quantidade de trabalho de cada pacote de trabalho. Ver figura 1 que representa os fluxos de trabalhos do processo descrito nesse tópico. Nesse processo, verificou-se que as modificações no projeto e na EAP demandam trabalho extra ao orçamentista para sincronizar as informações no cronograma e no orçamento. Os principais problemas encontrados foram: Grande volume de informação migrante entre as ferramentas utilizadas; Omissões e erros na leitura do projeto; A atividade manual que naturalmente exige mais tempo e é propensa a erros humanos; entre outros problemas também relatados na literatura $[6,7]$. 


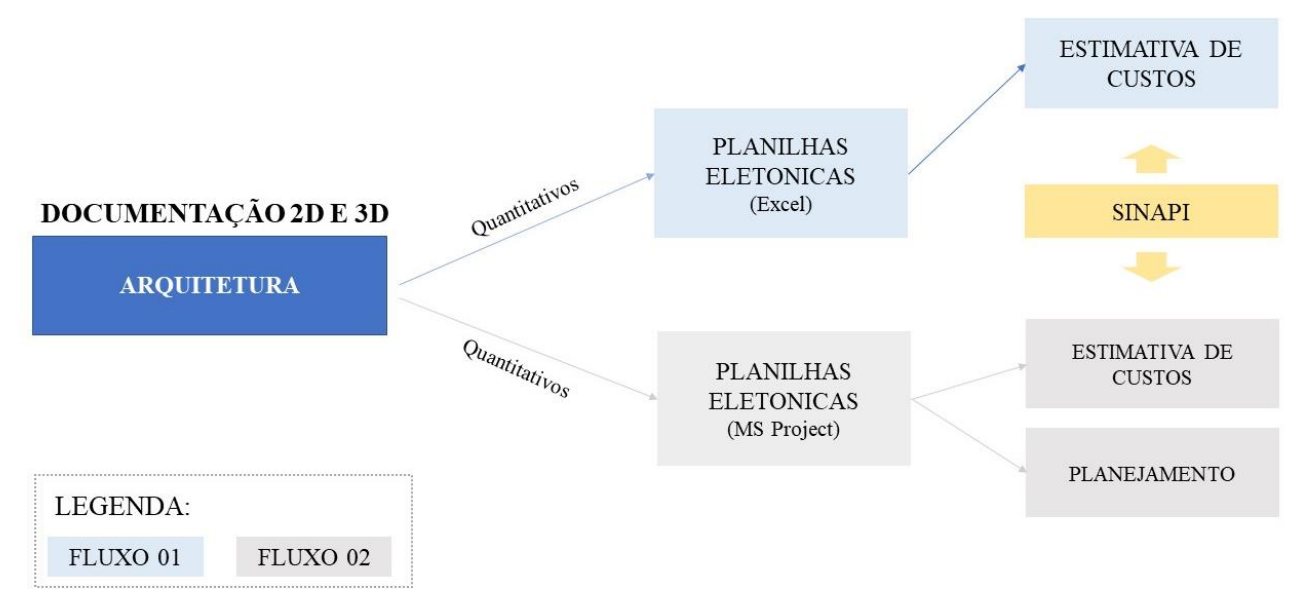

Figura 1: Fluxos do processo tradicional.

\section{Processo BIM}

Utilizando os modelos tridimensionais do Revit®, em sua configuração básica, foi possível realizar quantificações de áreas e volumes de paredes, pisos, lajes, coberturas entre outros elementos mais o levantamento de quantidade de portas, janelas, peças sanitárias, conexões etc. Tudo isso através das Tabelas de Quantidade e das Tabelas de Levantamento de Material que são nativas desse programa. Essas tabelas foram criadas em função das categorias de famílias existentes no protótipo virtual e permitiram a extração automática das informações.

Ressalta-se que durante esse processo, identificaram-se algumas limitações quanto ao acesso a informações tais como comprimentos e áreas de alguns materiais que não são computadas de acordo com os critérios de medição dos serviços a eles relacionados. Por exemplo, ao se quantificar a área de vidro das esquadrias em uma Tabela de Levantamento de Materiais, a informação apresentada é a área da superfície do sólido onde o material foi aplicado, de forma que esse dado não é a adequada ao critério de medição do pacote de trabalho. Para contornar esse contratempo, assim como outros, optou-se por desenvolver famílias de esquadrias e nelas inserir parâmetros compartilhados que possibilitaram o cálculo correto das quantidades.

Para alcançar uma melhor produtividade e organização durante o fluxo da informação, fez-se o uso de notas chaves (keynote) com o código do serviço e a sua descrição, uma orientação dada por um dos artigos da AU [5]. Por sua vez, essas notas deveriam estar associadas a cada material entre eles os existentes nas paredes, pisos, lajes entre outros. Com isso, foi possível criar tabelas conforme a EAP no próprio Revit@ e exportar os quantitativos para planilhas eletrônicas no Excel ${ }^{\circledR}$ e MS Project ${ }^{\circledR}$. Nesses softwares foi necessário inserir manualmente os preços de cada elemento, assim como ocorreu no processo tradicional, além de tratar e reunir dados das duas disciplinas (Arquitetura e Sistemas prediais hidrosanitários). No MS Project@ foi desenvolvido o sequenciamento das atividades, a rede de precedência e o encadeamento lógico dos serviços a serem executados no empreendimento, ou seja, o plano de execução da obra, além da estimativa de custo.

$\mathrm{O}$ arquivo com o plano de execução da obra mais os modelos BIM foram exportadas para o Navisworks ${ }^{\circledR}$ e reunidos em um único ambiente. Logo após, foi realizado a simulação do planejamento $4 \mathrm{D}$ e mais uma vez a extração dos quantitativos, que por sua vez revelou os 
mesmos resultados alcançados da ferramenta de modelagem. Ver figura 2 que representa os fluxos de trabalhos do processo descrito nesse tópico.

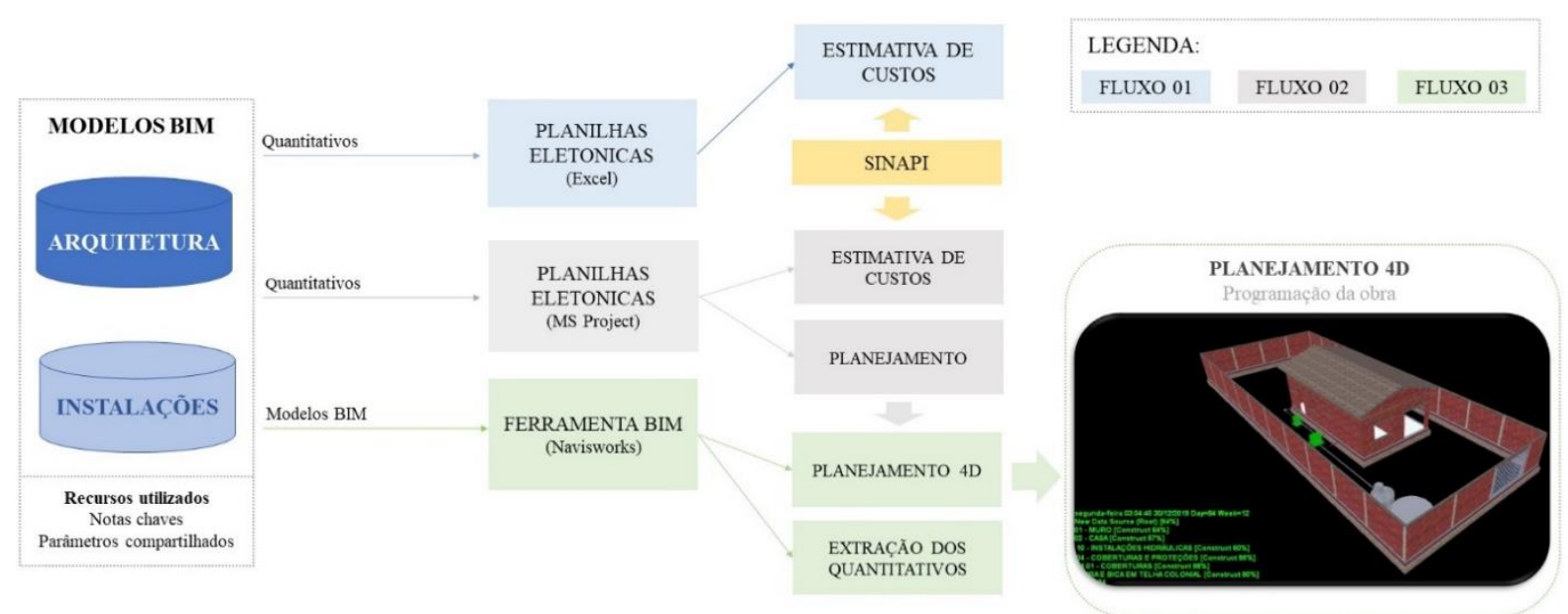

Figura 2: Fluxos do processo BIM.

Em síntese, nesse processo a quantificação é feita diretamente no modelo virtual de acordo com o nível de detalhamento, por meio de tabelas de quantidades de contagem de elementos ou de levantamento de materiais. Esses dados são exportados para planilhas eletrônicas e daí por diante esse processo BIM e o tradicional se assemelham, especialmente, no que diz respeito à determinação das composições de custo unitário, cálculo dos custos diretos de produção e elaboração do cronograma do empreendimento.

Observou-se que o modelo mal detalhado ou mal elaborado, assim como o desconhecimento da maneira com a ferramenta de modelagem BIM gera as quantidades, podem comprometer o processo de forma crítica. Essa perceção é apresentada também em outras pesquisas $[5,6,8]$. Soma-se a isso a dificuldade na ferramenta de modelagem, que foi utilizada, de sintetizar a informação em uma única tabela com todos os dados das diversas categorias com suas respetivas unidades e critérios de medição, bem como as quantidades efetivamente utilizadas. Outro ponto crítico desse processo é a excessiva quantidade de tarefas manuais, especialmente, na inserção de dados (EAP e Custo) nos componentes e na sua vinculação às tarefas no planejamento 4D. O excesso de ações manuais revelam que as atualizações são parcialmente automatizadas das estimativas de custo e prazo de execução em função de mudanças no escopo do projeto.

\section{Processo BIM com programação}

Utilizou-se recursos de programação tanto no ambiente de modelagem BIM como no ambiente das planilhas eletrônicas. A partir do Autodesk $®$ Dynamo® foram desenvolvidas rotinas para a ferramenta BIM utilizada. Uma dessas rotinas possibilitou a inserção de um parâmetro compartilhado chamado 4D_Task_ID, o qual armazena informações referentes à categoria, tipo, localização, fase e subfase de cada componente, condensando essas referências em um número único. Sendo esse composto ainda pelo próprio código da composição associada ao material que o constitui. Com esse parâmetro, que representa a EAP, foi possível a criação de 
uma tabela única dentro da ferramenta de modelagem. Com isso, nas planilhas eletrônicas não se fez mais necessário o tratamento dos dados, visto que as informações já estavam centralizadas e organizadas pela EAP. No software Navisworks ${ }^{\circledR}$ a criação dos conjuntos de elementos, os chamados Search Sets, foi facilitada pela utilização de um único critério de busca o parâmetro compartilhado 4D_Task_ID. Além disso, essa mesma referência passou a ser utilizada na vinculação das tarefas com os componentes no cronograma da obra, desse modo foi possível automatizar tanto a construção do cronograma como a sua atualização mediante a alterações no projeto.

Outra rotina do Dynamo® elaborada acede o banco de dados modelado no Microsoft ${ }^{\circledR}$ Access ${ }^{\circledR}$, que armazena todos os custos do SINAPI [4]. Desse modo, foi possível estimar o custo dos materiais e componentes no próprio ambiente de modelagem sem a necessidade de inserir o preço manualmente. Tal funcionalidade permite verificar o custo durante o desenvolvimento do modelo e tomar decisões sobre técnicas construtivas e especificações que viabilizem o empreendimento, algo fundamental nos estágios iniciais de desenvolvimento de qualquer empreendimento.

No ambiente das planilhas eletrônicas (Excel® e MS Project $\left.{ }^{\circledR}\right)$ foi criado, através de um script, um Suplemento, nomeado de Nostradamus ${ }^{\circledR}$, que acede também o banco de dados modelado no Access ${ }^{\circledR}$. Dessa maneira, foi possível a confeção de orçamentos em ambas as ferramentas, mas agora com a inserção do custo de modo automatizado. Outra funcionalidade existente no Suplemento Nostradamus ${ }^{\circledR}$ é a geração de um arquivo.XML. Esse arquivo pode ser importado pelo Navisworks ${ }^{\circledR}$ e com isso é viável criar os sets dos elementos conforme o parâmetro 4D_Task_ID, explicado anteriormente. Foram criados também rotinas e script que impulsionaram a interoperabilidade entre as ferramentas utilizadas. Ver figura 3 que representa o fluxo de trabalho do processo descrito nesse tópico.

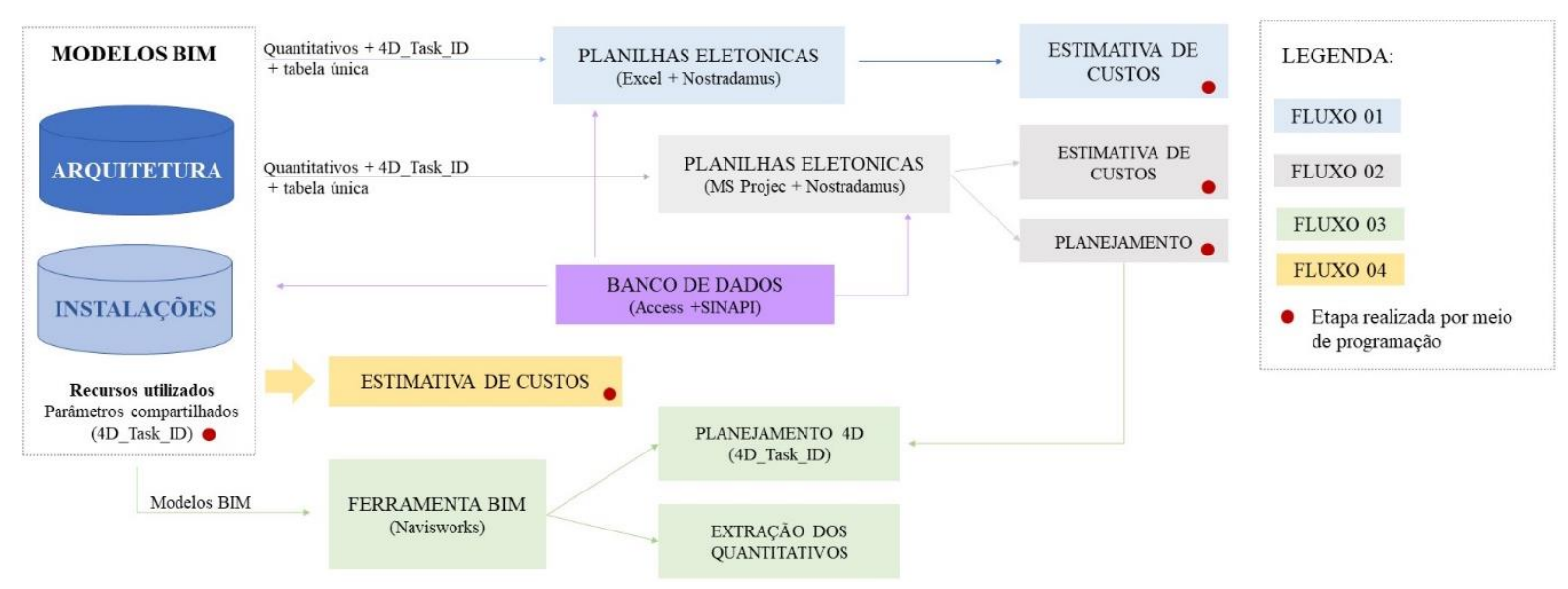

Figura 3: Fluxograma do processo BIM com programação.

No processo BIM com o uso de programação (script e visual) verificaram-se melhorias que mitigaram significativamente as dificuldades citadas anteriormente, especialmente no tocante à inserção repetitiva de informação nas diversas ferramentas. Além disso, obteve-se maior automatização na quantificação, na atualização da estimativa de custos e na elaboração do cronograma, quase que simultaneamente às atualizações do modelo virtual. Em suma, pode-se 
afirmar que o uso do BIM associado a programação apresenta ganhos significativos, também comprovados em outras publicações [1,9].

\section{Conclusão}

Um ponto comum nos três processos descritivos foi o uso da EAP e utilização da mesma referência de preço. Independente da ferramenta ou processo utilizado, a experiência do agente é fundamental, assim com a participação e colaboração de outros atores como os responsáveis pela execução, gerenciamento da obra e até mesmo os projetistas.

Comparados os dados obtidos na extração de quantitativos pode-se afirmar que ocorreu uma pequena variação e quando essa era investigada quase sempre indicava erros no procedimento tradicional oriundos de omissões do projeto e/ou falhas humanas. A maior diferença identificada entre os processos foi o tempo de extração e atualização e não nos valores em si, além da possibilidade de exibir tridimensionalmente as etapas da obra, o que permitiu fazer um planejamento mais eficiente e um cronograma mais assertivo. Computam-se ainda ganhos potenciais com a compatibilização e a apresentação da simulação da obra para clientes e equipe, facilitando uma melhor visualização e compreensão do empreendimento.

O objetivo deste trabalho foi descrever e analisar a aplicação de ferramentas BIM no processo de orçamentação e planejamento de obras. Em função da análise feita, constatou-se que a quantificação no processo tradicional é um trabalho árduo, intenso e propenso a erros. E que as funcionalidades nativas da ferramenta BIM utilizadas na pesquisa ainda implica na execução de diversas tarefas manuais que podem comprometer os resultados na orçamentação e no planejamento. Com a utilização da programação foi possível automatizar processos antes manuais, repetitivos e enfadonhos.

Apesar da perspetiva animadora que o último processo trouxe para os pesquisadores verificase a necessidade de mais estudos que aprimorem o processo BIM e as programações criadas. Para futuros trabalhos sugere-se investigar outras ferramentas BIM, em conjunto com recursos de programação, utilizar outros estudos de casos oriundos de diferentes usos e com mais disciplinas, investigar diretrizes que norteiam o processo de modelagem quando o propósito for a orçamentação e a programação da obra e investigar a gestão dessas informações em um ambiente comum de dados (CDE).

\section{Referências}

[1] Bezerra, C.R.M., (2019). Utilização de ferramenta BIM no gerenciamento de obras. Trabalho de conclusão de curso, Universidade Paulista, Pós-graduação em gerenciamento de obras e tecnologia da construção.

[2] Eastman C. M. , Teicholz P., Sacks R., and K. Liston, BIM handbook : a guide to building information modeling for owners, managers, designers, engineers, and contractors, 2nd Edition. New Jersey, EUA: John Wiley \& Sons, Inc., 2011

[3] Bezerra C.R.M., (2017). Extração de quantitativos e estimativas de custo com o uso da tecnologia BIM. Trabalho de conclusão de curso, Universidade Paulista, MBA em Plataforma BIM - Modelagem, Planejamento e Orçamento. 
[4] SINAPI. Referência de preços e custos. Disponpivel em: <http://www.caixa.gov.br/poderpublico/apoio-poder-publico/sinapi/Paginas/default.aspx> Acesso em: 02 de dezembro de 2019.

[5] Giesta J. P., E. Lira C. F. da S. Lira, Costa T. G., Silva J. P. J. A. A. da Silva. O uso da tecnologia BIM na quantificação e no planejamento: estudo de caso de residência RN- BR" in: 2. ${ }^{\circ}$ Congresso Português de Building Information Modelling (2018), Lisboa, Portugal, 2018, pp. 487-495. doi: 10.5281/zenodo. 1226766

[6] CHAGAS G.F.C., (2019). Análise comparativa entre fluxos de modelagem para orçamentação em BIM a partir da aplicação em uma residência multifamiliar situada no município de Natal/RN. Trabalho de conclusão de curso (Bacharelado em Engenharia Civil) - Universidade Federal do Rio Grande do Norte, Centro de Tecnologia, Curso de Engenharia Civil, Natal, RN, Disponível em: https://monografias.ufrn.br/jspui/handle/123456789/9980>. Acesso em: 2 mai. 2020

[7] Leusin S., Canellas R., Capistrano L. Integração entre o Revit, o orçamento e o planejamento de obra com uso de keynotes.Autodesk University Brasil 2012. Disponível em: <https://pt.scribd.com/document/342175404/AUBR-45-Apostila> Acesso em: 02 de junho de 2020.

[8] TRINDADE. L. D.; SANTOS.E. T. Definição de diretrizes de modelagem BIM para quantificação em diferentes etapas do processo de projeto. In: SIMPÓSIO BRASILEIRO DE TECNOLOGIA DA INFORMAÇÃO E COMUNICAÇÃO NA CONSTRUÇÃO E SIMPÓSIO BRASILEIRO DE GESTÃO E ECONOMIA DA CONSTRUÇÃO, 1 e 10. 2017, Fortaleza. Anais eletrônicos...Fortaleza. Disponível em: < https://marketingaumentado.com.br/sbtic/files/2017/paper_45.pdf >. Acesso em: 2 mai. 2020.

[9] J. Danado, "Fluxos de informação BIM - Recolha e partilha automatizada de dados a partir de um modelo BIM" in: 1. ${ }^{\circ}$ Congresso Português de Building Information Modelling (2016), Guimaraes, 2016, pp. 109-119. doi: 10.5281/zenodo.166758 